\title{
Modeling superlattice patterns using the interference of sharp focused spherical waves
}

\author{
Fidirko N.S
}

Samara State Aerospace University

\begin{abstract}
In this paper, modelling of pseudonondiffrational beams forming superlattice structures in a cross section has been performed. To create such distributions we suggest using superposition of sharp focused spherical waves. Thus, we have done simulations for several spherical waves generated by coherent light sources located on a ring with a certain radius. It is shown that depending on the configuration of the source field we can achieve different superlattice patterns in a cross section with a small amount of waves in the input field. Using more waves, we can obtain Bessel-like beams in the cross section.
\end{abstract}

Keywords: interference, optical vortices, sharp focusing, polarization

Citation: Fidirko NS. Modeling superlattice patterns using the interference of sharp focused spherical waves. Proceedings of Information Technology and Nanotechnology (ITNT-2015), CEUR Workshop Proceedings, 2015; 1490: 45 52. DOI: 10.18287/1613-0073-2015-1490-45-52

\section{Introduction}

A nondiffracting wave field is comprehended as a monochromatic optical field whose transverse shape remains invariant in free-space propagation. In 1987, Durnin proposed that nondiffracting wave fields are exact solutions to the homogeneous Helmholtz equation [1]; such particular solutions can be described as Bessel functions and are called nondiffracting Bessel beams. The realizable beams that propagate with relatively small divergence angles up to a certain range have finite energy and are known as pseudonondiffracting optical beams. Along with his co-authors, Durnin first experimentally realized a pseudonondiffracting Bessel beam in a cylindrical coordinates system [2]. Since then, nondiffracting Bessel beams have been extensively studied and applied in diverse fields, for example optical manipulation, the capture of micro particles and optical coherence tomography [3-7].

In recent years, the attention of physicists and mathematicians has been drawn to two-dimensional nondiffractive superlattice patterns [8]. Besides, realization of such distributions related to crystals, quasicrystals and other periodic structures has been actively researched [9-12]. 
A two dimensional distribution made by superposition of several plain lattices is called a superlattice $[8,12]$. In this work, we show an approach to create superlattice distributions using the interference of sharp focused spherical waves.

\section{Model of sharp focusing}

A sharp focused electromagnetic field in the focal area in Cartesian coordinates can be described with the following equation:

$$
\begin{aligned}
& \left(\begin{array}{l}
\mathbf{E}(\rho, \varphi, z) \\
\mathbf{H}(\rho, \varphi, z)
\end{array}\right)=-\frac{i f}{\lambda} \int_{0}^{\alpha} \int_{0}^{2 \pi} B(\theta, \phi) T(\theta)\left(\begin{array}{c}
\mathbf{P}_{E}(\theta, \phi) \\
\frac{1}{\mu} \mathbf{P}_{H}(\theta, \phi)
\end{array}\right) \times \\
& \times \exp [i k(\rho \sin \theta \cos (\phi-\varphi)+z \cos \theta)] \sin \theta d \theta d \phi,
\end{aligned}
$$

where $(\rho, \varphi, z)$ - cylindrical coordinates in the focal area, $(\theta, \phi)$ - spherical angular coordinates of the output pupil of the focusing system, $\alpha$ - maximal value of the azimuth angle, related to the numerical aperture, $B(\theta, \phi)$ - transmission function, $T(\theta)$ - apodization function (for aplanatic systems it is $T(\theta)=\sqrt{\cos \theta}$ ), $k=2 \pi / \lambda$ - wavenumber, $\lambda$ - wavelength, $f-$ focal distance. $\mathbf{P}_{E}(\theta, \phi)$ и $\mathbf{P}_{H}(\theta, \phi)$ - polarization matrixes for electric and magnetic fields respectively:

$$
\begin{aligned}
& \mathbf{P}_{E}(\theta, \phi)=\left[\begin{array}{cc}
1+\cos ^{2} \phi(\cos \theta-1) & \sin \phi \cos \phi(\cos \theta-1) \\
\sin \phi \cos \phi(\cos \theta-1) & 1+\sin ^{2} \phi(\cos \theta-1) \\
-\sin \theta \cos \phi & -\sin \theta \sin \phi
\end{array}\right]\left(\begin{array}{l}
c_{x}(\phi) \\
c_{y}(\phi)
\end{array}\right) ; \\
& \mathbf{P}_{H}(\theta, \phi)=\left[\begin{array}{cc}
\sin \phi \cos \phi(\cos \theta-1) & -\left(1+\cos ^{2} \phi(\cos \theta-1)\right) \\
1+\sin ^{2} \phi(\cos \theta-1) & -\sin \phi \cos \phi(\cos \theta-1) \\
-\sin \theta \sin \phi & \sin \theta \cos \phi
\end{array}\right]\left(\begin{array}{l}
c_{x}(\phi) \\
c_{y}(\phi)
\end{array}\right) .
\end{aligned}
$$

where $c_{x}(\phi), c_{y}(\phi)$ is the polarization coefficients of the source field.

For vortex fields $B(\theta, \phi)=R(\theta) \exp (i m \phi)$, so formula (1) can be reduced to an equation with one-time integration:

$$
\left(\begin{array}{c}
\mathbf{E}(\rho, \varphi, z) \\
\mathbf{H}(\rho, \varphi, z)
\end{array}\right)=-i k f \int_{0}^{\alpha} R(\theta) T(\theta)\left(\begin{array}{c}
\mathbf{Q}_{E}(\rho, \varphi, \theta) \\
\frac{1}{\mu} \mathbf{Q}_{H}(\rho, \varphi, \theta)
\end{array}\right) \sin \theta \exp (i k z \cos \theta) \mathrm{d} \theta .
$$

where $\mathbf{Q}_{E, H}(\rho, \varphi, \theta)$ matrices can be explicitly written for certain types of polarizations and consist of superposition of Bessel functions of different orders [1314].

If all beams are generated by different zones of the optical element supplementing a lens with a high numerical aperture, the resulting field in the focal area will be a superposition of the fields established by different zones of the optical element: 


$$
\mathbf{E}(\rho, \varphi, z)=\sum_{i} \mathbf{E}_{i}(\rho, \varphi, z)
$$

\section{The interference of spherical waves}

The next step is to review an opaque diaphragm, imposed on the pupil of a focusing system with a high numerical aperture. The diaphragm has several small holes, located evenly on a certain radius from the centre of the diaphragm.

Table 1. - The resulting electric field for the central radius $r_{c}=25 \lambda$

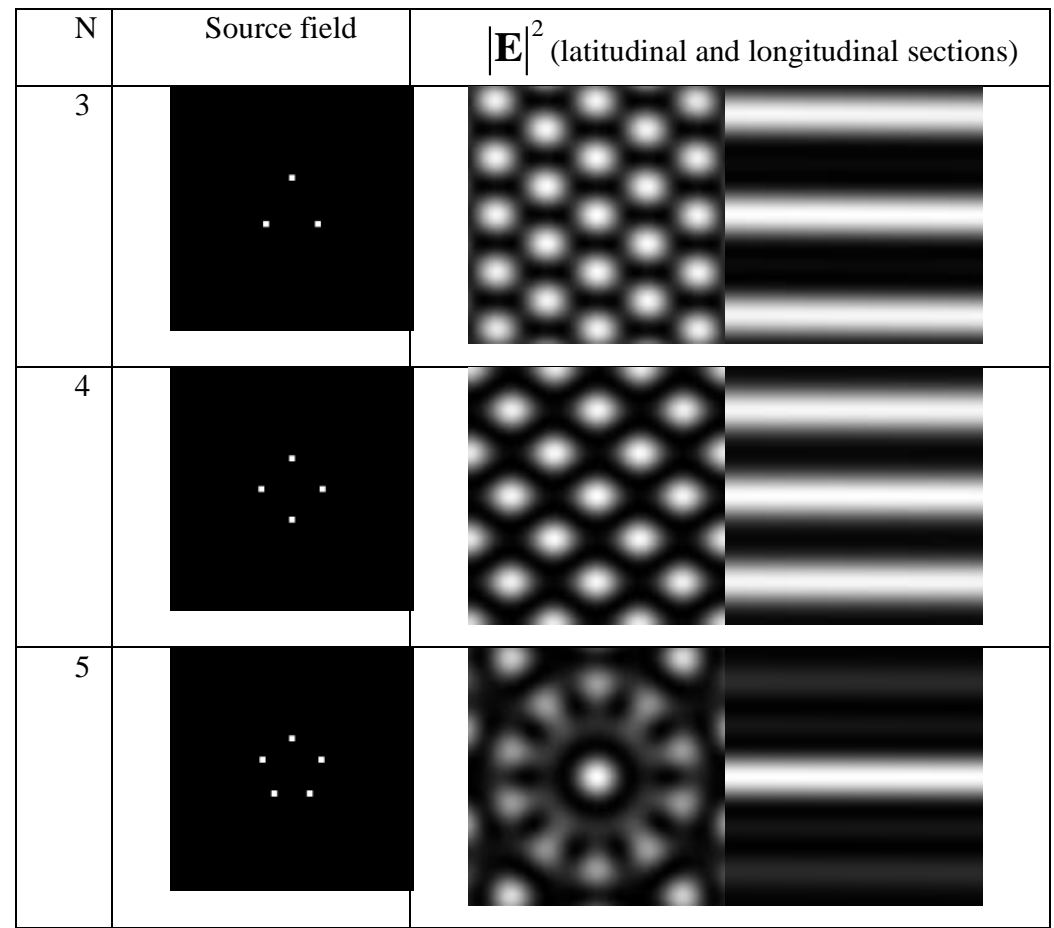

Thereby, we gain a system of point light sources. Every source generates a spherical wave that is being focused on and interferes with waves from other point sources. Herewith, we can vary the number of point sources and their distance from the centre of the aperture.

In figure 1, the shapes of the diaphragms generating different number of waves is evident. In tables 1-5 results of modelling with different parameters are listed.

From the tables above, with the interference of three and four spherical waves in the cross section of the focal area there are bright light spots located in the lattice sites; furthermore, the configurations of the lattices can be different. In addition, longitudinal plane long light channels are formed. The nondiffractional nature of the beams, the spectrum of which is localized on the ring, has been evident for a long time. It has been successfully used to create different structures that remain invariant 
in the longitudinal direction [16-18]. The obtained distributions can be used to create photonic crystals and plasma channels.

Table 2. - The resulting electric field for the central radius $r_{c}=50 \lambda$

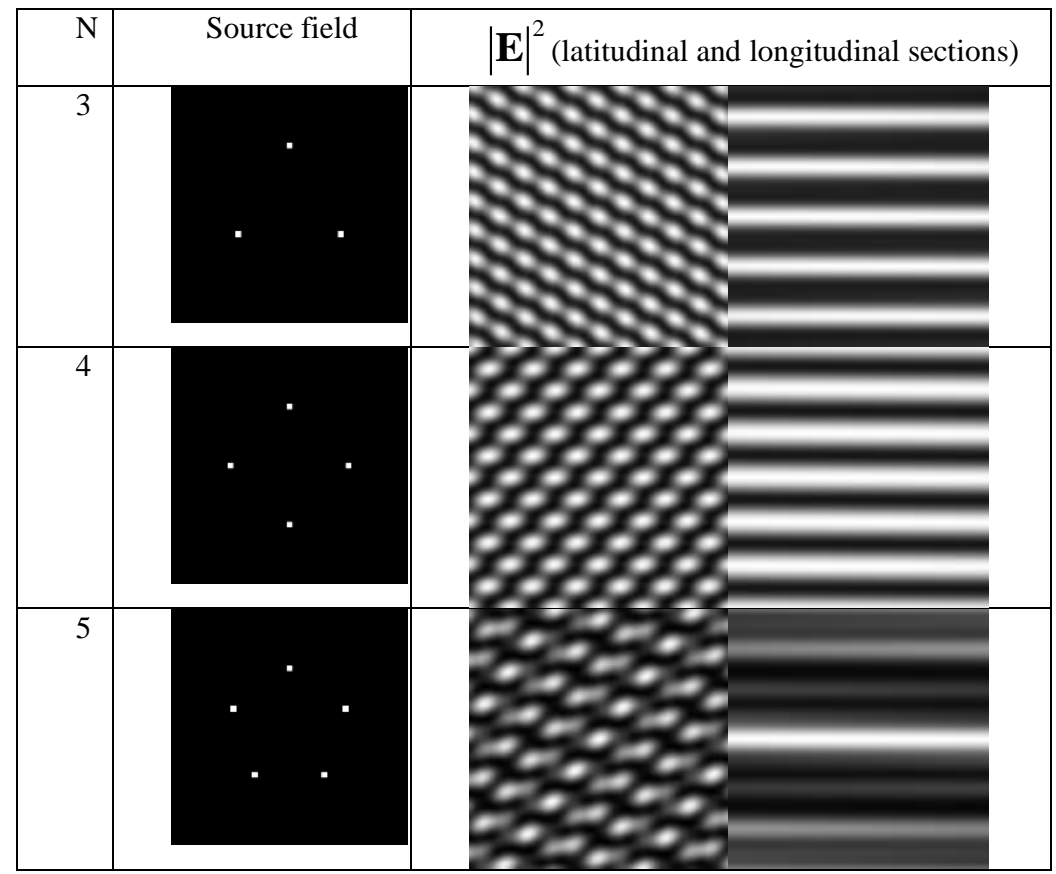

With the interference of five or more waves in the cross section, we can see a superlattice pattern. If the radius of the ring is increased (for comparison see tables 3 and 4) the central spot is decreased, which corresponds to the increase in the numerical aperture. With the increase in the numerical aperture (radius of the ring), the interference pattern becomes more complex and different symmetries appear.
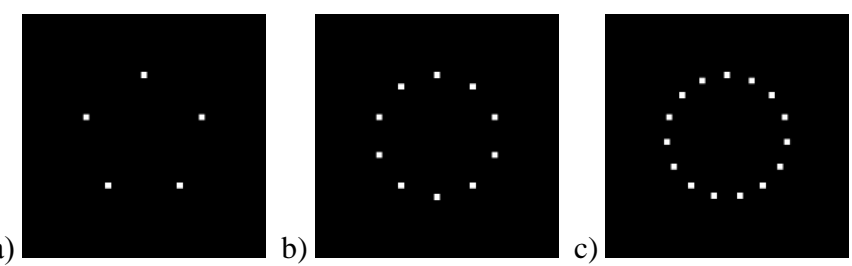

Fig. 1. - Shape of the diaphragm for a) 5 point sources, b) 10 point sources, c) 15 point sources with a central radius $r_{c}=50 \lambda$, the radius of every point is $-r_{d}=2 \lambda$ 
Table 3. - The resulting electric field for the central radius $r_{c}=25 \lambda$

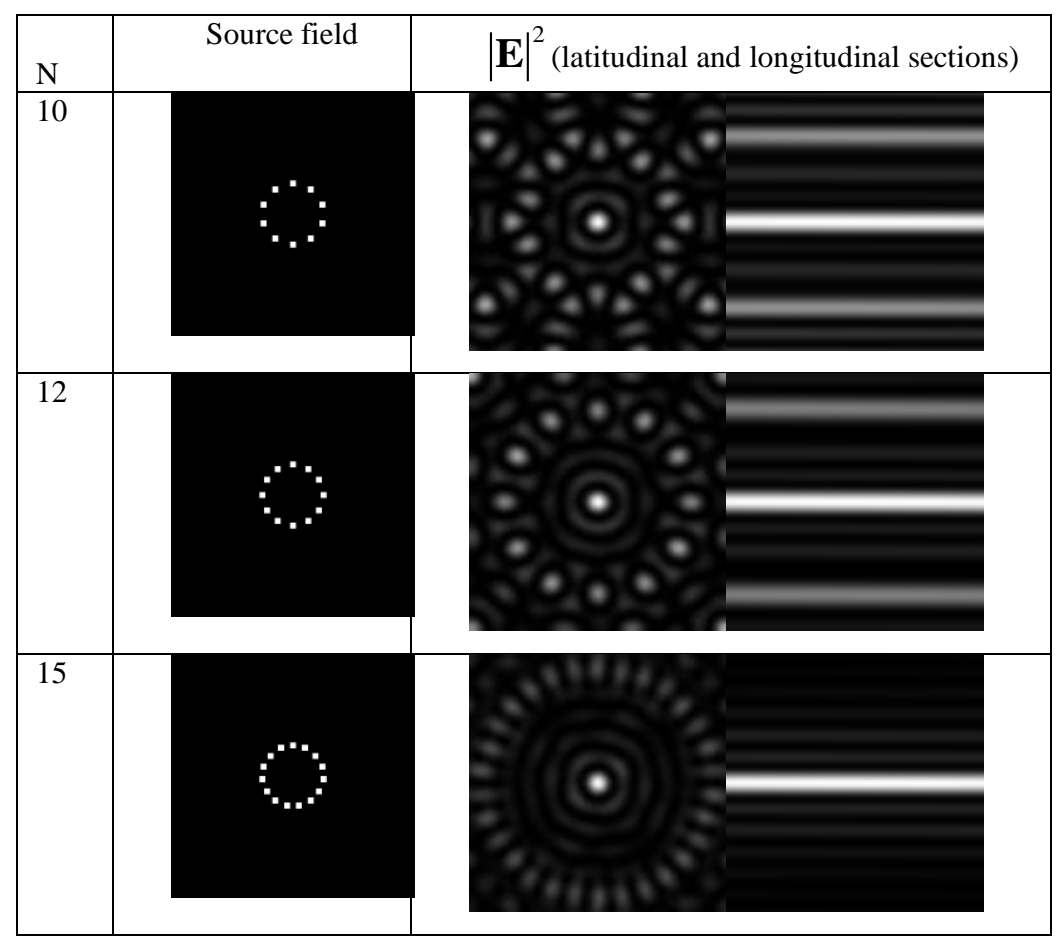

If we use many point sources so that the ring aperture is tightly filled, Bessel-like beams begin to form in the cross section. In these situations, the size of the central spot of the Bessel beam depends on the radius of the ring aperture. It is worth noting that this approach to generating a Bessel beam is more convenient than creating the ring aperture.

\section{Conslusion}

By varying the number of waves and distance between them one can therefore obtain a wide range of superlattice patterns in the cross section, which will keep their structure at a long distance. In this case, the radius of the holes in the diaphragm and the radius of the ring determine the length of the longitudinal section. Increasing the size of the holes and the numerical aperture leads to a reduction of the focus depth.

More complex superlattice patterns can be added by increasing the number of phases of the point sources with special phase elements and by adding polarization to the focused beam [13, 19-21].

\section{Acknowledgements}

This work was financially supported by the Russian Ministry of Education and Science. 
Table 4. - The resulting electric field for the central radius $r_{c}=50 \lambda$

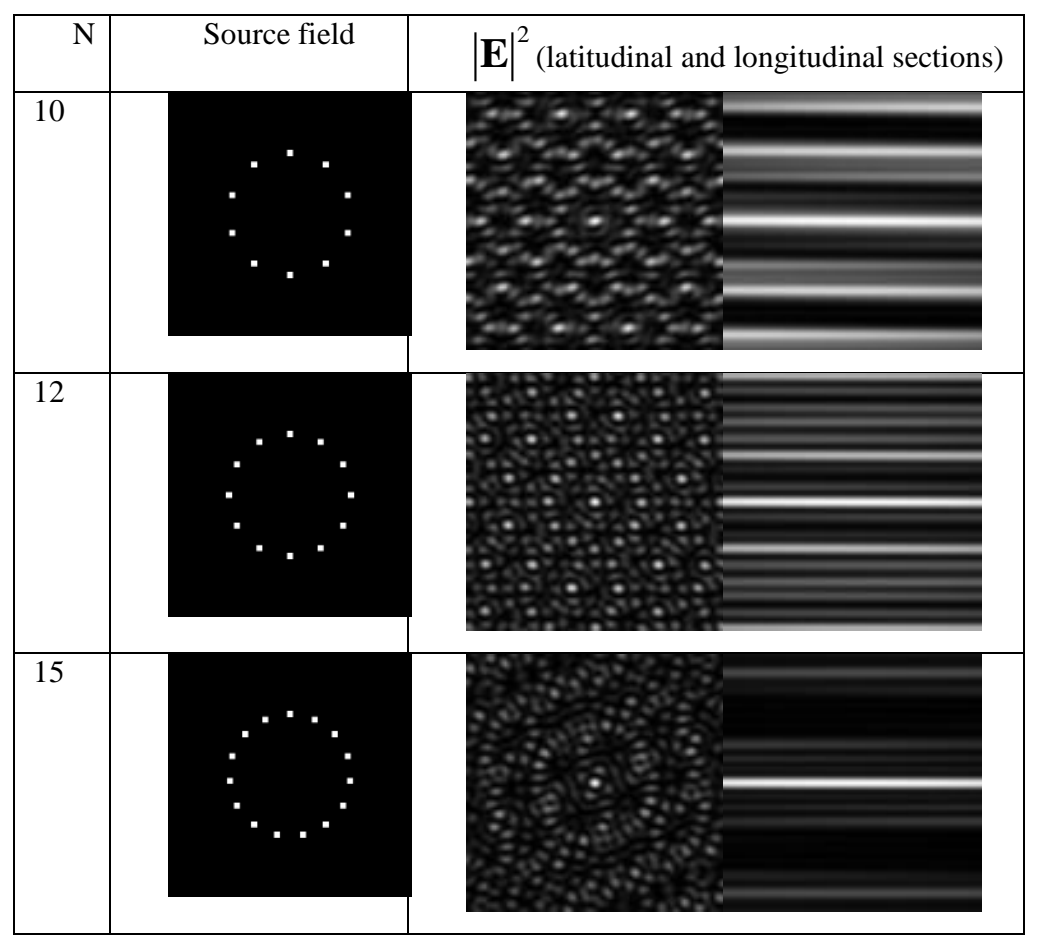

\section{References}

1. Durnin J. Exact solutions for nondiffracting beams. I. The scalar theory. Journal of the Optical Society of America A, 1987; 4(4): 651-654.

2. Durnin J, Miceli JJ Jr, Eberly JH. Diffraction-free beams. Physical Review Letters, 1987; 58(15): 1499-1501.

3. Garcés-Chávez V, McGloin D, Melville H, Sibbett W, Dholakia K. Simultaneous micromanipulation in multiple planes using a self-reconstructing light beam. Nature, 2002; 419(6903): 145-147.

4. McGloin D, Garcés-Chávez V, Dholakia K. Interfering Bessel beams for optical micromanipulation. Optics Letters, 2003; 28(8): 657-659.

5. Arlt J, Garcés-Chávez $\mathbf{V}$, Sibbett $\mathbf{W}$, Dholakia K. Optical micromanipulation using a Bessel light beam. Optics Communications, 2001; 197(4-6): 239-245.

6. Ding Z, Ren H, Zhao Y, Nelson JS, Chen Z. High-resolution optical coherence tomography over a large depth range with an axicon lens. Optics Letters, 2002; 27(4): 243245.

7. Yu C, Wang MR, Varela AJ, Chen B. High-density non-diffracting beam array for optical interconnection. Optics Letters, 2000; 177(1-6): 369-376.

8. Bouchal Z. Nondiffracting optical beams: physical properties, experiments, and applications. Czechoslovak Journal of Physics, 2003; 53(7): 537-624.

9. Boguslawski M, Rose P, Denz C. Increasing the structural variety of discrete nondiffracting wave fields. Physical Review A, 2011; 84(1): 013832. 
Table 5. - The resulting electric field for the central radius $r_{c}=75 \lambda$

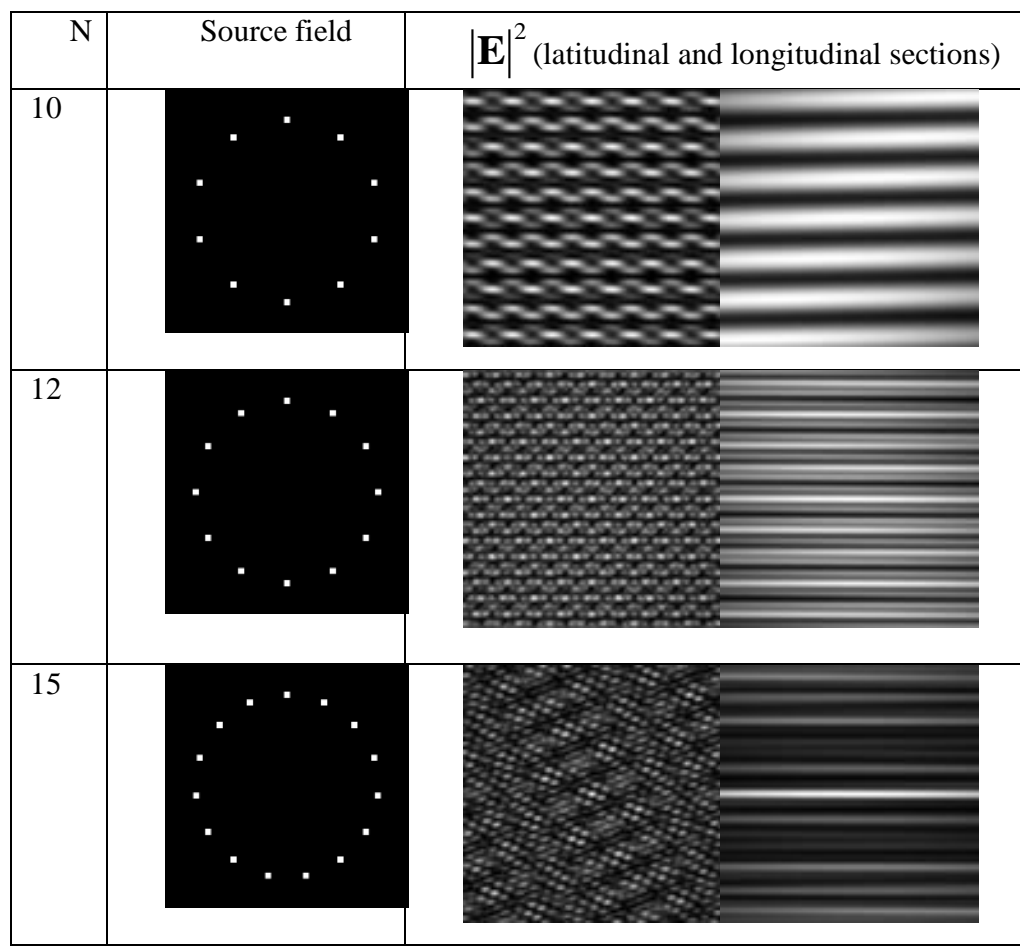

10. Boguslawski M, Rose P, Denz C. Nondiffracting kagome lattice. Applied Physics Letters, 2011; 98(6): 061111.

11. Chen YF, Liang HC, Lin YC, Tzeng YS, Su KW, Huang KF. Generation of optical crystals and quasicrystal beams: Kaleidoscopic patterns and phase singularity. Physical Review A, 2011; 83(5): 053813.

12. Tsou CH, Wu TW, Tung JC, Liang HC, Tuan PH, Chen YF. Generation of pseudonondiffracting optical beams with superlattice structures. Optics Express, 2013; 21(20): 23441-23449.

13. Khonina SN, Volotovsky SG. Managing the contribution of various vector field components in the focal area of a high-aperture lens with binary phase structures. Computer Optics, 2010; 34(1): 58-68. [in Russian]

14. Khonina SN, Kazanskiy NL, Volotovsky SG. Vortex phase transmission function as a factor to reduce the focal spot of high-aperture focusing system. Journal of Modern Optics, 2011; 58(9): 748-760.

15. Khonina SN, Kazanskiy NL, Volotovsky SG. Influence of vortex transmission phase function on intensity distribution in the focal area of high-aperture focusing system. Optical Memory and Neural Networks (Information Optics), 2011; 20(1): 23-42.

16. Ziolkowski RW, Besieris IM, Shaarawi AM. Aperture realizations of exact solutions to homogeneous-wave equations. Journal of the Optical Society of America A, 1993; 10(1): 75-87.

17. Gutierrez-Vega JC, Iturbe-Castillo MD, Chavez-Cerda S. Alternative formulation for invariant optical fields: Mathieu beams. Optics Letters, 2000; 25(20): 1493-1495. 
18. Khonina SN. An easy and efficient way to create different nondiffractional laser beams. Computer Optics, 2009; 33(1): 70-78. [in Russian]

19. Gao X, Wang J, Gu H, Xu W. Focusing properties of concentric piecewise cylindrical vector beam. Optik, 2007; 118: 257-265.

20. Khonina SN, Golub I. Optimization of focusing of linearly polarized light. Optics Letters, 2011; 36(3): 352-354.

21. Khonina SN, Fidirko NS. Research of the interference of counterpropagating sharp focused beams with different polarizations. News of the Samara Science Center of the RAS, 2014; 16(4): 27-33. [in Russian]

22. Khonina SN, Ustinov AV. Analysis of interference of cylindrical laser beams generated by ring optical elements with a vortex phase at sharp focusing. Computer Optics, 2015; 39(1): 12-25. [in Russian] 